\title{
Los profesores de matemáticas y la articulación de sentidos asignados a representaciones semióticas en situaciones de tratamiento. El caso de la probabilidad simple
}

\author{
Gladys Mejía Osorio \\ Pedro Javier Rojas Garzón
}

\begin{abstract}
Resumen: Se presentan resultados parciales de una investigación sobre dificultades que encuentran profesores de matemáticas para articular sentidos asignados a representaciones semióticas obtenidas mediante tratamiento, específicamente relacionadas con una tarea de probabilidad: "lanzar un dado y obtener un número par", analizada con herramientas del Enfoque Ontosemiótico (EOS), la cual se desarrolló en el marco del Doctorado en Educación de la Universidad Francisco José de Caldas (Colombia). Se realizó desde un estudio de caso, a partir de entrevistas basadas en tareas. Se evidenció que los profesores calculan correctamente la probabilidad pedida, reconocen que ésta puede representarse mediante diferentes expresiones aritméticas (equivalencia sintáctica); sin embargo, no reconocen la equivalencia semántica entre dichas expresiones; adicionalmente, estos resultados son similares a los reportados en investigaciones previas realizadas con estudiantes.
\end{abstract}

Palabras clave: Función semiótica. Configuraciones cognitivas. Articulación de sentidos. Tratamiento.

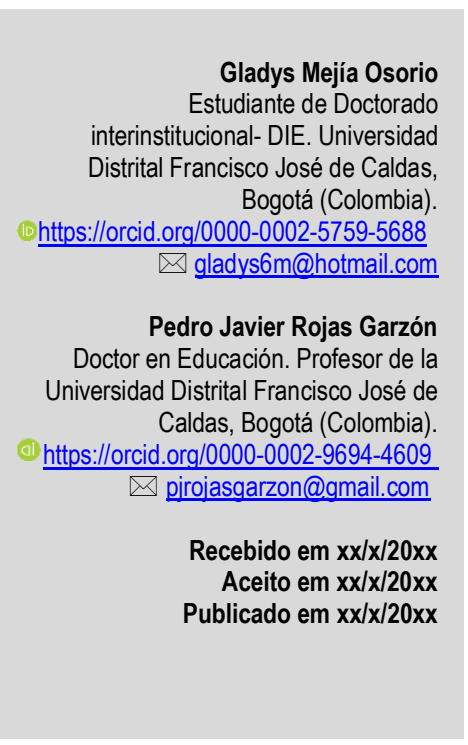

\section{Professores de matemática e a articulação dos sentidos atribuídos às representações semióticas em situações de tratamento. 0 caso da probabilidade simples}

Resumo: São apresentados resultados parciais de uma investigação sobre as dificuldades encontradas pelos professores de matemática para articular os significados atribuídos às representações semióticas obtidas através do tratamento, especificamente relacionadas com uma tarefa de probabilidade: "jogar um dado e obter um número par", analisada com as ferramentas do Approach Ontosemiotic (EOS), que se desenvolveu no marco do Doutorado em Educação da Universidade Francisco José de Caldas (Colômbia). Foi conduzido a partir de um estudo de caso, com base em entrevistas baseadas em tarefas. Constatou-se que os professores calculam corretamente a probabilidade solicitada, eles reconhecem que ela pode ser representada por diferentes expressões aritméticas (equivalência sintática); no entanto, eles não reconhecem a equivalência semântica entre essas expressões; além disso, esses resultados são semelhantes aos relatados em pesquisas anteriores realizadas com alunos.

Palavras-chave: Função semiótica. Configurações cognitivas. Articulação dos sentidos. Tratamento.

\section{Teachers of mathematics and the articulation of senses assigned to semiotic representations in treatment situations. The case of simple probability}

Abstract: Partial results of an investigation are presented on difficulties encountered by mathematics
teachers to articulate meanings assigned to semiotic representations obtained through treatment, 
specifically related to a probability task: "throwing a dice and obtaining an even number", analyzed with the Approach tools Ontosemiotic (EOS), which was developed within the framework of the Doctorate in Education at the Francisco José de Caldas University (Colombia). It was conducted from a case study, based on task-based interviews. It was evidenced that the teachers correctly calculate the requested probability, they recognize that it can be represented by different arithmetic expressions (syntactic equivalence); however, they do not recognize the semantic equivalence between these expressions; additionally, these results are similar to those reported in previous research carried out with students.

Keywords: Semiotic function. Cognitive configurations. Articulation of senses. Treatment.

\section{Introducción}

En los procesos de enseñanza y aprendizaje de las matemáticas es fundamental que los sujetos recurran a una variedad de sistemas semióticos de representación o registros de representación puesto que "los objetos matemáticos no son perceptibles directamente por los sujetos sino vía las representaciones semióticas que permiten denotarlos y a su vez posibilitan una manipulación sobre estos” (DUVAL, 2004, p. 17). Por esta razón, en el área de matemáticas se requiere que tanto profesores como estudiantes reconozcan un mismo objeto desde diferentes representaciones y que éstas sean apropiadas para expresar y representar ideas matemáticas, así como transformarlas para formular y sustentar distintos puntos de vista que requieren del dominio de diversos recursos y registros semióticos, tanto en lenguaje natural como matemático. Los profesores, en particular, requieren de un conocimiento didáctico, disciplinar y pedagógico que les posibilite orientar adecuadamente actividades específicas que potencien la apropiación y el desarrollo de dichas competencias matemáticas por parte de sus estudiantes. En esta dirección, este estudio centra la atención en el conocimiento disciplinar de los profesores de matemáticas, e indaga por las dificultades que éstos encuentran para relacionar entre sí los sentidos asignados a representaciones semióticas de un mismo objeto matemático obtenidas mediante tratamiento, relación que Rojas (2012) ha denominado articulación de sentidos o articulación semiótica.

Se presentan los significados asignados a las representaciones semióticas que emergen en las producciones realizadas por 11 profesores ( 5 de primaria y 6 de secundaria) a una tarea específica, seleccionados de una población total de 64 profesores, se reportan posibles causas de dificultad para relacionar los sentidos asignados entre sí. Se contrastan los resultados con los hallazgos reportados por Rojas $(2012,2015)$ frente al trabajo con estudiantes al resolver la misma tarea.

Se analiza la posibilidad de realizar una articulación semiótica utilizando herramientas suministradas por el Enfoque Ontosemiótico del Conocimiento y la Instrucción Matemática (EOS), puesto que este enfoque, por un lado, adopta una concepción pragmática de significado y, por otro, permite identificar las prácticas matemáticas de los profesores vinculadas a los sentidos 
asignados por ellos a representaciones semióticas obtenidas mediante tratamiento, así como las relaciones que establecen por medio de las funciones semióticas que emergen en el proceso de significación. Las funciones semióticas son un constructo teórico empleado en diferentes investigaciones con el fin de analizar procesos de significación de objetos matemáticos (DISTÉFANO; AZNAR y POCHULU, 2012; ROJAS, 2012; DISTÉFANO; POCHULU y FONT, 2015; DISTÉFANO y POCHULU, 2017).

\section{Antecedentes de investigación}

D’Amore (2006) plantea que no sólo las transformaciones de conversión entre representaciones semióticas son objeto de dificultades para la comprensión de las matemáticas sino también las de tratamiento, en tanto generan cambios en los sentidos asignados a éstas 0 , en términos de Rojas $(2012,2015)$ no articulación semiótica. Para soportar dicha tesis, se han realizado algunas investigaciones que documentan el fenómeno de la no articulación semiótica, en las cuales se reportan evidencias de las dificultades que encuentran algunos estudiantes para articular los sentidos asignados a dos expresiones reconocidas sintácticamente equivalentes, obtenidas mediante tratamiento, puesto que, logran aplicar las transformaciones requeridas al interior de un registro de representación para obtener una de ellas a partir de la otra, pero no admiten que éstas son semánticamente equivalentes.

Santi (2011) reportó resultados obtenidos en un experimento de enseñanza con 12 estudiantes de 19 años de edad de último año de estudios de secundaria en una escuela de Bolonia (Italia), y muestra que los estudiantes se enfrentan a tres "juegos lingüísticos" diferentes que están detrás del sistema de prácticas y configuraciones de objetos que manejan: El primero, de la geometría euclidiana; en este contexto, el concepto de tangente (como entidad primaria) se define como la línea recta perpendicular al rayo en un punto de la circunferencia. El segundo, el concepto de tangente a una cónica (como una entidad primaria) se define como la línea recta. El tercero, concepto de tangente (como un objeto primario) se define como el paso de la línea recta a través del punto tangente del gráfico de la función cuya pendiente es la derivada de la función en el punto tangente. El autor aporta evidencias de dificultades tanto en el proceso cognitivo de conversión como también en el de tratamiento y plantea que una dificultad para asignarle sentido al concepto de tangente se liga con el trabajo por separado de cada uno de los juegos del lenguaje, es decir, al trabajo fragmentado de los conceptos que se relacionan con este objeto matemático. Reconoce que es necesario centrarse en la actividad reflexiva, como el análisis de los signos, las 
representaciones, las prácticas compartidas, y los elementos semióticos que generan dificultades en la comprensión del objeto.

Por su parte, Rojas (2012) documentó el fenómeno relacionado con las dificultades que encuentran los estudiantes para articular los sentidos asignados a representaciones semióticas de un mismo objeto matemático obtenidas mediante tratamiento, por medio, de la descripción y análisis de los procesos de asignación de sentidos de 9 estudiantes, 6 de grado $9^{\circ}$ de educación básica y 3 de grado $11^{\circ}$, en 3 instituciones de Bogotá (Colombia), en relación con 3 tareas específicas (de probabilidad simple, de expresiones algebraicas y de geometría analítica). El autor indagó el sentido asignado a representaciones semióticas obtenidas por tratamiento. Reporta que los estudiantes encuentran dificultades para articular los sentidos asignados a expresiones asociadas con un mismo objeto matemático, algunos reconocen la equivalencia sintáctica entre dos o más expresiones dadas, a partir de una de las expresiones logran realizar las transformaciones de tratamiento requeridas para obtener otra expresión, pero no siempre consiguen articular los sentidos asignados a estas (no reconocen la equivalencia semántica) e incluso pueden cambiar el sentido inicialmente asignado a éstas.

Rojas (2012) plantea que algunas dificultades que encuentran los estudiantes para articular los sentidos asignados a expresiones pueden concentrarse en cuatro grupos: (i) reconocimiento icónico de las expresiones, en tanto asignan significado a las expresiones basados en un reconocimiento icónico de las mismas; (ii) anclaje a situaciones dadas, puesto que, se evidencia una tendencia a realizar interpretaciones ligadas con la situación propuesta, por ejemplo, al pedir calcular la probabilidad que al lanzar un dado se obtenga un número par; (iii) interacción y cambios en la interpretación; (iv) falta de apropiación del lenguaje matemático.

En el presente trabajo se asume que en toda transformación semiótica de tratamiento subyace la noción de equivalencia, al realizar un rastreo de la literatura existente sobre dicha noción, se evidencia que ésta ha sido abordada básicamente en dos tópicos: conjuntos numéricos y álgebra. En el contexto numérico la equivalencia aparece en los primeros años escolares durante el desarrollo del concepto de número, la ampliación del sistema numérico decimal y las relaciones que existe entre los distintos tipos de números; en esta dirección, la equivalencia aparece como necesaria para relacionar distintas representaciones de los números, reconocer relaciones entre sus operaciones y resolver cierto tipo de problemas. Por ejemplo, una visión relacional del signo igual posibilita que los estudiantes tengan una comprensión de algunos objetos matemáticos, como en el caso del trabajo con ecuaciones algebraicas, que requiere una visión del signo igual 
como una relación entre dos cantidades, o en la resolución de una ecuación que conserva la relación de equivalencia.

En el contexto algebraico se estudian las relaciones entre diferentes tipos de expresiones, aplicando reglas de transformación de expresiones (perspectiva sintáctica) o mediante la compresión del empleo de métodos numéricos (perspectiva semántica). Desde la perspectiva sintáctica establecen relaciones y reglas inherentes al álgebra, privilegiando el lenguaje simbólico, aspectos procedimentales y las reglas de transformación de expresiones para probar la equivalencia; desde la perspectiva semántica, resaltan la interpretación del contexto para establecer la equivalencia de expresiones (KIERAN y SALDANHA, 2005; KIERAN; BOILEAU; TANGUAY y DRIJVERS, 2013; SOLARES y KIERAN, 2013; CHALÉ-CAN; FONT y ACUÑA, 2017). Al respecto, Booth (1989, como se citó en RADFORD, 2004) plantea que desde la perspectiva semántica no puede obviarse la perspectiva semántica, puesto que la habilidad para manipular símbolos algebraicos requiere aplicar propiedades estructurales de las operaciones y otras relaciones matemáticas, que deben ser comprendidas.

Estas dos maneras de enfocar la equivalencia (sintáctica-semántica) son tenidas en cuenta por Mejías (2019) quien realizó una investigación sobre los conocimientos relacionados con la enseñanza del álgebra, evaluando específicamente los conocimientos didácticos y matemáticos que poseen los profesores de matemáticas de educación primaria en Chile respecto al álgebra temprana. Las tareas propuestas, en su mayoría, tenían que ver con la equivalencia de expresiones algebraicas; por ejemplo, debían inferir si la afirmación "Sumo tres números consecutivos. Si divido el resultado por tres, obtengo siempre el segundo número", formulada por un estudiante, era o no válida.

Mejías (2019) alude que entre las producciones que indicaban que la conjetura era válida para todos los números enteros se hallan respuestas en las que prima la perspectiva semántica y otras en las que prima la perspectiva sintáctica. Las que se consideraron como parcialmente correctas, el autor reportó que algunos profesores recurren a la autoconsciencia de no saber aplicar las propiedades requeridas para dicha comprobación (perspectiva sintáctica) realizan afirmaciones como la siguiente: "Sí, teóricamente no tengo idea porqué, pero si pruebo con los números da". Resultados que permiten corroborar que para el estudio y desarrollo de la noción de equivalencia algebraica es necesario tener en cuenta elementos sintácticos y semánticos de las expresiones algebraicas, conectados y desarrollados conjuntamente para promover una mejor comprensión de dicha noción (SOLARES y KIERAN, 2013; KIERAN; BOILEAU; TANGUAY y DRIJVERS, 2013; CHALÉ-CAN et al., 2017). 
La experiencia previa de los estudiantes con las transformaciones algebraicas, relacionadas con procesos reiterativos de factorización, expansión, agrupación y simplificación de términos, permite desarrollar una noción espontánea de equivalencia, no siempre consciente, que implica la vinculación de expresiones por medio de estas transformaciones (perspectiva sintáctica). Así, se sugiere la necesidad de una transición desde una noción espontánea de la equivalencia hacia una concepción que esté más asociada con el aspecto semántico, además del reconocimiento explícito de las propiedades aplicadas para realizar las transformaciones requeridas (KIERAN et al., 2013).

Por su parte Chalé-Can (2018) investigó el papel que juegan los aspectos sintácticos y semánticos en la equivalencia de expresiones algebraicas en secuencias visuales, encontrando que se hace fundamental relacionar dichos aspectos en el aula para una mejor comprensión de la equivalencia algebraica; resaltando la necesidad de un proceso de significación que dé sentido a los símbolos y posibilite un proceso de generalización que permita enunciar una proposición como punto de partida para el tratamiento simbólico posterior. Chalé-Can et al., (2017) consideran que la mayoría de los sujetos realizan una evaluación numérica que les permite tener un acercamiento semántico de la equivalencia posibilitando un desarrollo primitivo e ingenuo de la noción de equivalencia, hecho que permite una vinculación de expresiones por medio de diferentes transformaciones como factorización, expansión, agrupación y simplificación de términos.

Si bien el tratamiento numérico permite que los profesores admitan y tengan un acercamiento a la equivalencia, no posibilita que algunos reconozcan la igualdad entre las expresiones desde el aspecto semántico; lo cual muestra la importancia que tiene la articulación de los elementos sintácticos y semánticos en la equivalencia de expresiones dadas, puesto que posibilita una mejor comprensión de dicho constructo (SOLARES y KIERAN, 2013; KIERAN et al., 2013). Por otro lado, en la literatura existente son pocas las investigaciones que giran en torno a la transformación semiótica de tratamiento, específicamente aquellos en el que se documentan las posibles dificultades que encuentran los sujetos para articular sentidos a representaciones semióticas obtenidas mediante tratamiento. Partiendo de ello, en el presente trabajo se pretende mostrar evidencias del fenómeno de la no articulación de sentidos o semiótica en los profesores de matemáticas en ejercicio, se parte del supuesto que los elementos sintácticos y semánticos son parte constitutiva de la noción de equivalencia (CHALÉ-CAN et al., 2017). 
El análisis de las producciones de profesores que se reporta en esta investigación se realizó usando instrumentos del Enfoque Ontosemiótico del Conocimiento y la Instrucción Matemática (EOS), desde este enfoque se considera un objeto matemático como todo aquello que es indicado, señalado o nombrado cuando se hace, se comunica o se aprende matemáticas (GODINO, 2002). La práctica matemática es asumida como toda actuación o expresión (verbal, gráfica, etc.) realizada por una persona (o compartida en el seno de una institución) para resolver problemas matemáticos, comunicar, validar o generalizar la solución a estos problemas (GODINO; CASTRO; AKÉ y WILHELMI, 2012). En la práctica matemática se activa una configuración de objetos primarios que describen seis tipos de objetos primarios (FONT; GODINO y GALLARDO, 2013) a saber: (1) el Lenguaje (términos, expresiones, gráficos, etc.); (2) los Conceptos (mediante definiciones o descripciones); (3) las Proposiciones (enunciados sobre conceptos); (4) los Procedimientos (algoritmos, operaciones, técnicas, etc.); (5) las Situaciones (problemas, tareas, ejercicios, etc.); y (6) los Argumentos (validan las proposiciones y procedimientos).

Las situaciones problemas son el origen y motivación de la actividad, el lenguaje es el soporte para representar a las demás entidades y son un instrumento para la acción, los argumentos justifican tanto los procedimientos como las proposiciones que, ligadas a las definiciones, resuelven las situaciones-problemas. Tal y como se ilustra en la Figura 1 que muestra las interrelaciones entre los objetos primarios que, a su vez, se relacionan con los símbolos del lenguaje (significantes).

Figura 1. Configuración de objetos primarios

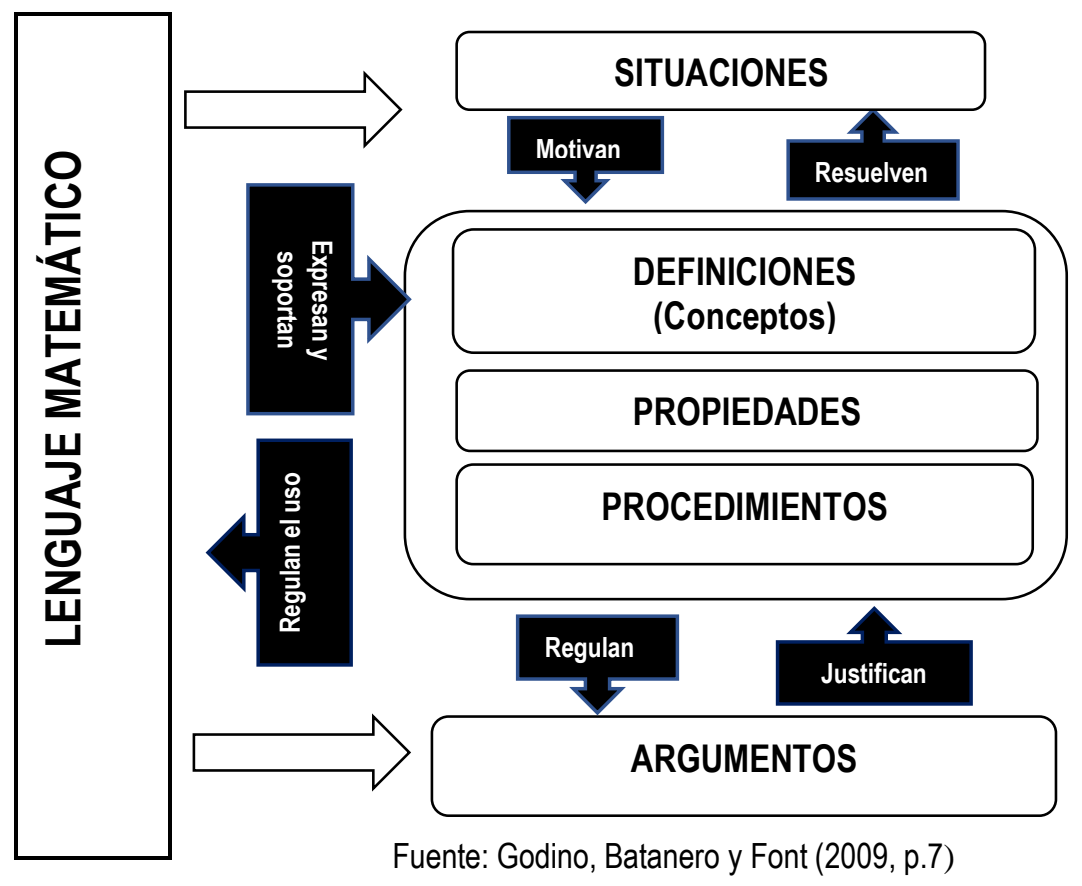


Estas relaciones entre los seis objetos primarios determinan las configuraciones, definidas por Godino, Batanero y Font $(2007$, p.8) como "las redes de objetos intervinientes y emergentes de los sistemas de prácticas y las relaciones que se establecen entre los mismos", que son activadas por los sujetos, que a su vez son herramientas teóricas para describir los conocimientos matemáticos, en su doble versión, personal e institucional. Los objetos matemáticos que intervienen en las prácticas matemáticas y los emergentes de las mismas, según el juego de lenguaje en que participan, pueden ser considerados desde las siguientes facetas o dimensiones duales: personal - institucional; ostensivo - no ostensivo; expresión - contenido; extensivo intensivo (ejemplar - tipo); y unitario - sistémico (GODINO, 2002).

En concordancia con Godino, Batanero, Burgos y Gea (2021) el análisis que se presenta en este estudio se centró en la faceta expresión-contenido que, por un lado, permitió evidenciar y comprender las relaciones de los distintos sentidos que los profesores otorgan a las representaciones que se relacionan y emergen en cada una de las tareas y, por otro, tomar una posición frente a la articulación y no articulación de sentidos en términos de funciones semióticas. Con base en los planteamientos de Rojas (2012) se asume la articulación semiótica como el proceso de encadenamiento semiótico en el que a una misma expresión/antecedente se le asignan dos contenidos/consecuente diferentes que se relacionan entre sí, de manera análoga se asumió la no articulación semiótica como el proceso interrumpido de encadenamiento semiótico en el que a una misma expresión/antecedente se le asignan dos contenidos/consecuente diferentes que no se logran relacionar entre sí. Además, se comparte con este autor que el sentido de un objeto es el contenido que tiene al objeto primario como antecedente/expresión de la función semiótica.

Tabla 1. Sentido asignado a un objeto matemático primario

\begin{tabular}{|c|c|}
\hline Antecedente /Expresión & Consecuente/contenido \\
\hline Objeto primario & Sentido del Objeto primario \\
\hline
\end{tabular}

Fuente: Rojas (2012, p. 51)

Por otro lado, Rojas (2012) plantea que un mismo objeto primario puede tener diferentes sentidos. Por ejemplo, en la tarea del cálculo de la probabilidad: "obtener un número par al lanzar un dado", los resolutores pueden calcular la probabilidad del evento aleatorio recurriendo a diferentes expresiones matemáticas, que a su vez participan en un proceso de semiosis ${ }^{1}$.

\footnotetext{
1 Para Peirce los signos son empleados por los sujetos para formar nuevas ideas y nuevos conceptos en un proceso denominado semiosis.
} 
Tabla 2. Diferentes sentidos de un objeto, que institucionalmente se espera construyan los aprendices

\begin{tabular}{|c|c|}
\hline $\begin{array}{c}\text { Antecedentel } \\
\text { Expresión }\end{array}$ & $\begin{array}{c}\text { Consecuentel } \\
\text { Contenido }\end{array}$ \\
\hline$\frac{3}{6}$ & $\begin{array}{c}\text { Casos favorables sobre } \\
\text { casos posibles }\end{array}$ \\
\hline
\end{tabular}

\begin{tabular}{|c|c|}
\hline $\begin{array}{c}\text { Antecedentel } \\
\text { Expresión }\end{array}$ & $\begin{array}{c}\text { Consecuentel } \\
\text { Contenido }\end{array}$ \\
\hline 0.5 & $\begin{array}{c}\text { El cociente entre dos } \\
\text { números } a \text { y } b .\end{array}$ \\
\hline
\end{tabular}

\begin{tabular}{|c|c|}
\hline $\begin{array}{c}\text { Antecedentel } \\
\text { Expresión }\end{array}$ & $\begin{array}{c}\text { Consecuentel } \\
\text { Contenido }\end{array}$ \\
\hline$\frac{1}{2}$ & $\begin{array}{c}\text { La mitad de números } \\
\text { pares e impares }\end{array}$ \\
\hline
\end{tabular}

\begin{tabular}{|c|c|}
\hline $\begin{array}{c}\text { Antecedentel } \\
\text { Expresión }\end{array}$ & $\begin{array}{c}\text { Consecuentel } \\
\text { Contenido }\end{array}$ \\
\hline $50 \%$ & $\begin{array}{c}\text { La mitad de casos } \\
\text { favorables }\end{array}$ \\
\hline
\end{tabular}

Fuente: Adaptado de Rojas (2012, p. 52)

En los ejemplos anteriores se relacionan algunas expresiones matemáticas a las que pueden recurrir los sujetos para representar la probabilidad pedida. Según Rojas (2012) frente a los diferentes sentidos de objeto matemático primario se produce una articulación de sentidos cuando se establece una función semiótica entre dos sentidos diferentes de un mismo objeto matemático primario, es decir, cuando uno de los sentidos (consecuente/ contenido) del objeto primario se convierte en antecedente/expresión de una nueva función semiótica que tiene como consecuente/contenido a otro sentido de dicho objeto. Por ejemplo, el siguiente caso se muestra la relación de dos sentidos diferentes de un mismo objeto matemático.

Tabla 3. Ejemplo de una función semiótica

\begin{tabular}{|c|c|c|}
\hline \multicolumn{2}{|c|}{ Antecedente/ Expresión } & Consecuente/Contenido \\
\hline $\begin{array}{c}\text { Antecedentel } \\
\text { Expresión }\end{array}$ & $\begin{array}{c}\text { Consecuentel } \\
\text { Contenido }\end{array}$ & $\begin{array}{c}1 / 2 \text {, la mitad de casos } \\
\text { favorables. (Atributo de la } \\
\text { probabilidad pedida) }\end{array}$ \\
\cline { 1 - 2 }$\frac{3}{6}$ & $\begin{array}{c}\text { Casos favorables sobre } \\
\text { casos posibles }\end{array}$ & Sentido 2 \\
\hline
\end{tabular}

Fuente: Adaptado de Rojas (2012, p.52)

En el ejemplo anterior se relacionan dos sentidos diferentes "casos favorables sobre casos posibles" "la mitad de casos favorables" que corresponden al consecuente/contenido de la nueva función semiótica que un sujeto puede establecer. Ambos casos corresponden a los sentidos que le pueden asignar a la expresión 3/6. En este ejemplo, se tiene un objeto matemático primario, que le son asignados dos sentidos diferentes. La concatenación de funciones semióticas se puede reducir a una sola función semiótica que pone en relación (articulación semiótica) los dos sentidos, como se muestra en el siguiente ejemplo: 
Tabla 4. Ejemplo de una función semiótica como articulación de sentidos

\begin{tabular}{|c|c|}
\hline $\begin{array}{c}\text { Antecedentel } \\
\text { Expresión }\end{array}$ & $\begin{array}{c}\text { Consecuentel } \\
\text { Contenido }\end{array}$ \\
\hline $\begin{array}{c}\text { Casos favorables sobre } \\
\text { casos posibles }\end{array}$ & $\begin{array}{c}1 / 2 \text {, la mitad de casos favorables } \\
\text { (Atributo de la probabilidad pedida) }\end{array}$ \\
\hline
\end{tabular}

Fuente: Adaptado de Rojas (2012, p.52)

En palabras de Rojas (2012) la articulación es el resultado de la concatenación de dos funciones semióticas, es decir, se origina una articulación de sentidos, cuando se establece una nueva función semiótica en la que una de las dos funciones semióticas anteriores juega el papel de antecedente/expresión. En el ejemplo del cálculo de la probabilidad el aprendiz puede recurrir a cualquier expresión matemática que exprese la mitad de los casos (equivalencia de fracciones). Por otro lado, este autor alude que en la articulación semiótica el consecuente/contenido de una función que se consideraban en un inicio no era relacionadas ahora son consideradas como equivalentes. En esta dirección de funciones semióticas el tratamiento puede ser caracterizado como una función semiótica, puesto que, dos objetos primarios son considerados como equivalentes en tanto ambos son representaciones de un mismo objeto matemático. Por ejemplo, mediante transformaciones semióticas en el registro lenguaje numérico 3/6, al aplicar los procedimientos necesarios, es equivalente a $1 / 2$ y a $4 / 8$, son sintácticamente equivalentes, originando una nueva función semiótica:

Tabla 5. Transformación tipo tratamiento. Equivalencia entre fracciones

\begin{tabular}{|c|c|}
\hline $\begin{array}{c}\text { Antecedentel } \\
\text { Expresión }\end{array}$ & Consecuente/ Contenido \\
\hline$\frac{3}{6}$ & $\frac{1}{2}=\frac{4}{8}=0.5=50 \%$ \\
Todas las expresiones equivalentes representan la mitad \\
de casos favorables.
\end{tabular}

Fuente: Adaptado de Rojas (2012, p.52)

Una vez establecida la equivalencia sintáctica de las expresiones (un objeto primario con dos representaciones), puesto que, una de ellas es obtenida a partir de otra como resultado de un proceso de tratamiento, se puede asociar el mismo sentido (el mismo consecuente/contenido); en este caso, al asignar el mismo consecuente/contenido a dos objetos primarios, se origina una articulación de sentidos. Se dice que no se reconoce una articulación semiótica cuando el sentido asignado a una representación no se articula con el sentido asignado posteriormente a otra representación obtenida de la primera mediante tratamiento, y las representaciones son asociadas con objetos matemáticos o situaciones diferentes (ROJAS, 2012). 
Por otro lado, en correspondencia con el EOS, se asumió que en la noción de equivalencia se encuentran presentes dos fuentes de significados parciales: el sintáctico y el semántico, así como su necesaria articulación, que brinda elementos para establecer que dos expresiones son equivalentes más allá de la aplicación de reglas. Se describen los significados personales de los profesores en términos de prácticas, configuraciones cognitivas de objetos primarios y procesos activados en estas prácticas y se identifican las diferentes relaciones que establecen por medio de las funciones semióticas.

Desde su aspecto sintáctico, se dice que dos expresiones son equivalentes cuando estas expresiones tienen una reescritura numérica o algebraica común, la cual puede ser obtenida por medio de la aplicación de propiedades algebraicas conocidas (conmutativa, asociativa, distributiva, identidades notables, simplificación, etc.). Desde su aspecto semántico, se dice que dos expresiones son equivalentes cuando se tiene un contexto en el que los símbolos de las expresiones adquieren significados contextuales y en el que, además, se puede decir que estos dos significados son el mismo. Tal y como se muestra en la Figura 2.

Figura 2. Equivalencia entre fracciones.

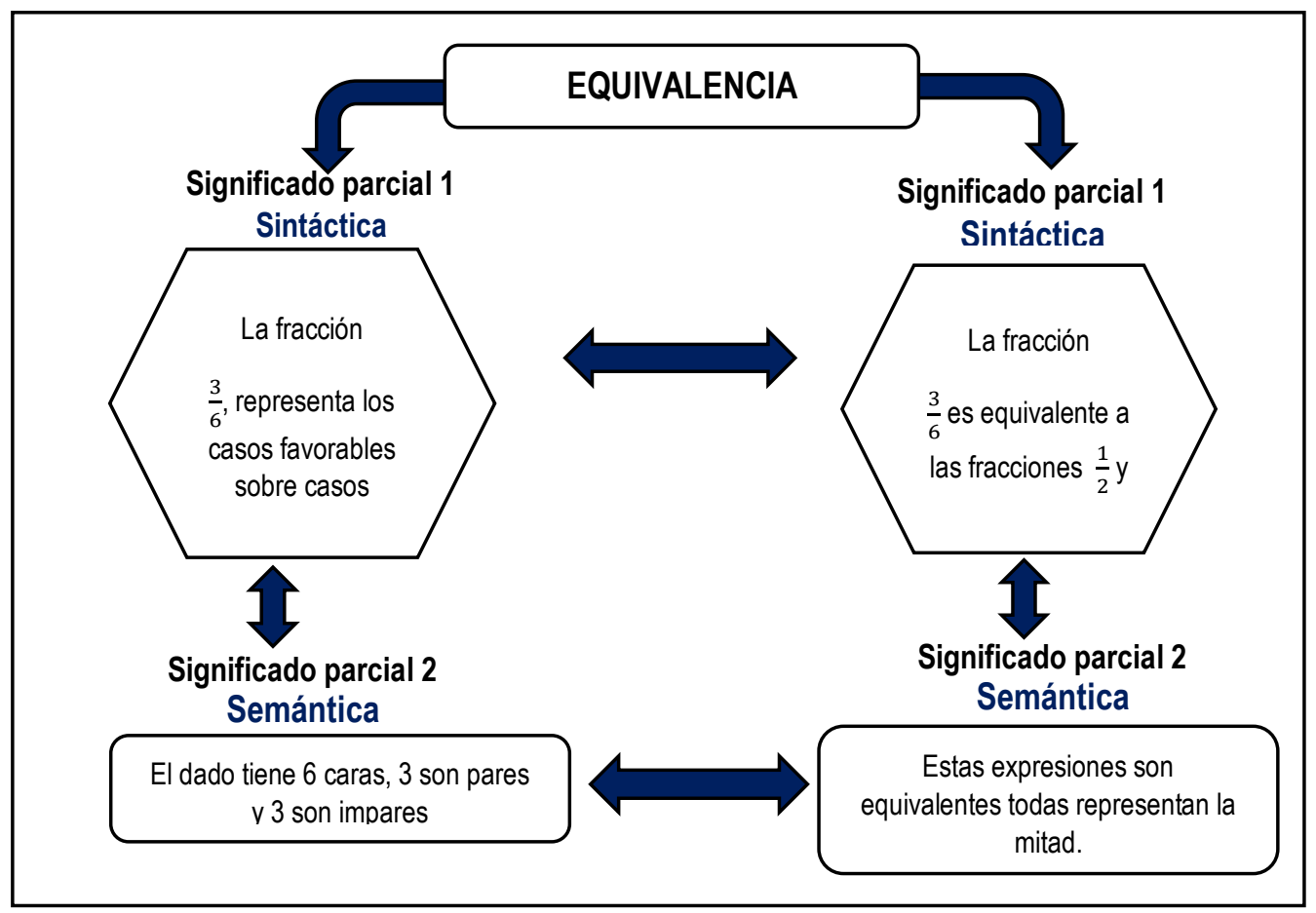

Fuente: Elaboración propia.

En el caso particular de la tarea propuesta, los profesores calculan la probabilidad pedida, recurren a expresiones matemáticas como $3 / 6,1 / 2,50 \%$, ó, 0.5. Reconocen que las fracciones $3 / 6,1 / 2$ y $4 / 8$ son equivalentes (sintácticamente); y cualquier fracción equivalente a $1 / 2$ es 
una fracción representativa de la probabilidad, debido que representa la mitad de casos favorables (equivalencia semántica).

Los profesores generalmente expresan la probabilidad por medio de la fracción 3/6, establecen una correspondencia entre los casos favorables sobre casos posibles que corresponde a la definición clásica de la probabilidad, aspecto que le permite aceptar la expresión $1 / 2$, como una manera de representar la probabilidad pedida, puesto que es una expresión que representa las dos opciones existentes (pares-impares), y cualquier fracción equivalente a 1/2, representaría la probabilidad pedida; no obstante, para varios profesores la fracción 4/8, no expresa la probabilidad pedida, en tanto no es una "expresión representativa" del dado (tradicional de 6 caras) pues el dado no tiene 8 caras.

\section{$4 \quad$ Metodología}

Este trabajo se enmarca en un enfoque de investigación cualitativa, de tipo descriptivo e interpretativo, con el propósito de analizar los sentidos asignados por un grupo de profesores de matemáticas en ejercicio a representaciones semióticas, y las dificultades que encuentra para relacionar los sentidos entre sí, es decir, para establecer una articulación semiótica (ROJAS, 2012, 2015). En este estudio se describe la construcción de significados personales por parte de profesores de matemáticas en situaciones que requieren transformaciones semióticas de tratamiento, y se realiza una caracterización de rasgos fundamentales en el proceso de significación de dichas representaciones. En tanto el fenómeno de la "articulación de sentidos" o "articulación semiótica" tiene múltiples variables, entre ellas la formación matemática de los profesores matemáticas que incide en el lenguaje matemático utilizado, los signos empleados y los significados que otorgan a estos, las representaciones movilizadas y las que moviliza en la enseñanza y aprendizaje de las matemáticas, los significados personales otorgados a los diferentes objetos matemáticos, la visión epistemológica y ontológica de las matemáticas, etc., para esta investigación se optó por un estudio de caso colectivo (STAKE, 1994).

Se contó con una población inicial de estudio de 64 profesores en ejercicio² (32 de educación primaria y 32 de educación secundaria), de los cuales se seleccionaron aquellos profesores que mínimo, en tres tareas de las cuatro propuestas, aplicaron una serie de procedimientos y reglas matemáticas (tratamientos) que les permitía reconocer la equivalencia sintáctica de expresiones,

\footnotetext{
2 La población que hace parte de este estudio se desempeña en educación básica (primaria y secundaria) y media vocacional, ubicados en 8 regiones de Colombia (Atlántico, Magdalena, Huila, Antioquia, Guajira, Bogotá, D.C "urbana-rural" y Cundinamarca).
} 
pero no relacionaban entre sí los sentidos o significados de dichas expresiones, lo cual impide que se establezca una articulación semiótica.

Se realizaron entrevistas semiestructuradas, basadas en tres tareas, a los 11 profesores que conforman el estudio de caso colectivo para encontrar posibles similitudes o diferencias en los argumentos dados con respecto al trabajo realizado por estudiantes a estas mismas tareas, con base en el estudio realizado por Rojas (2012) entre ellas la tarea sobre el cálculo de la probabilidad, de la cual se reportan en este documento los análisis y resultados:

(Tarea - Calculo de la probabilidad). En lo que sigue, siempre se hará referencia a un dado tradicional de seis caras.

1. ¿Cuál es la probabilidad de que lanzando un dado se obtenga un número par?

(a) La probabilidad es:

(b) Explique brevemente cómo realizó el cálculo de la probabilidad:

2. ¿La anterior probabilidad se podría representar con la expresión $1 / 2$ ?

(a) Marque con una equis $(X)$ su respuesta Sí ( ) No ( )

(b) Justifique a continuación.

3. ¿La probabilidad se podría representar con la expresión $4 / 8$ ?
(a) Marque con una equis $(X)$ su respuesta

(b) Justifique a continuación.

Para el análisis de las producciones de estos 11 profesores se hizo uso de instrumentos del EOS, Godino (2002) se identifica en las soluciones las prácticas matemáticas desarrolladas por ellos, las configuraciones cognitivas y relaciones que establecen por medio de funciones semióticas, se considera que estos elementos permiten describir y caracterizar de manera sistemática los objetos matemáticos primarios que interviene en la práctica matemática (situaciones/problemas, elementos lingüísticos, procedimientos, conceptos/definiciones, proposiciones/propiedades y argumentos), relacionados en las configuraciones cognitivas activadas, con el fin identificar y caracterizar las dificultades que encuentran los profesores para articular los sentidos asignados a representaciones semióticas obtenidas mediante tratamiento.

En el caso particular de esta tarea se seleccionaron aquellos profesores que calcularon de manera correcta la probabilidad pedida, recurriendo a expresiones matemáticas como 1/2,3/6, $0.50,50 \%$, reconocieron que dicha probabilidad podía ser representada por medio de la fracción $1 / 2$ y que la expresión 4/8 es equivalente, pero afirmaban que no es una "fracción representativa del dado", en tanto, el dado no tiene 8 caras sino 6 . En la siguiente producción que corresponde a la producción del profesor de educación secundaria-D, relacionada con la tarea antes referida, se evidencia que no establece la articulación semiótica requerida: 
Figura 3. ¿La probabilidad se podría representar con la expresión $4 / 8$ ?

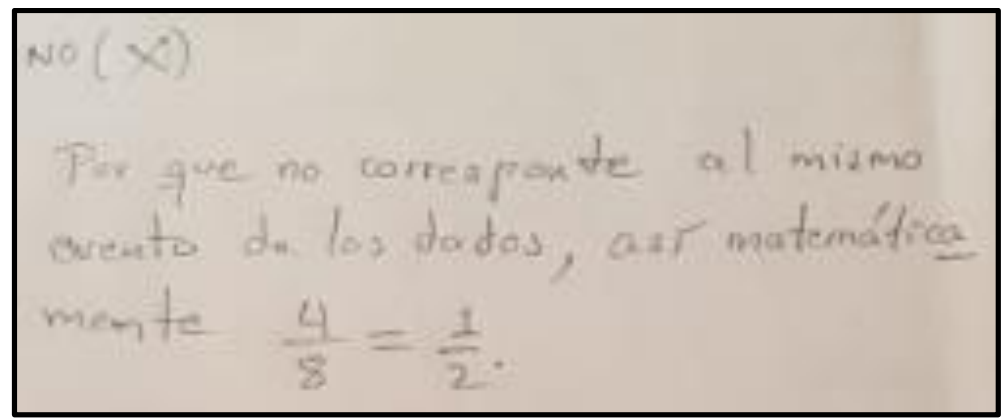

Fuente: Producción realizada por el profesor de secundaria- D.

El profesor afirma que 4/8 no representa la probabilidad pedida y argumenta que "No, porque no corresponde al mismo evento de los dados, así matemáticamente $4 / 8=1 / 2$ " Si bien reconoce la equivalencia sintáctica entre $3 / 6$ y 4/8, no reconoce la equivalencia semántica, en tanto, se genera un "anclaje" al objeto de la situación inicial, el "dado tradicional" de 6 caras (ROJAS, 2012, 2015). Con base a este criterio, se presentan los resultados con respecto a una de las 4 tareas propuestas, del grupo de 5 profesores de primaria, conformado por: primaria-A, -B, $C,-D,-E$, y 6 profesores de secundaria, conformado por: secundaria $-A-B-C-D$ y $-E$.

\section{$5 \quad$ Resultados}

Con el propósito de evidenciar los sentidos otorgados por los profesores a las representaciones semióticas que emergen en la tarea del cálculo de la probabilidad, así como establecer las dificultades que encuentran para relacionar los sentidos otorgados a éstas, se presenta una rejilla en la cual se sintetizan elementos básicos de las configuraciones cognitivas de objetos primarios activados. En la tabla 6 se presentan las configuraciones activadas por los profesores de Educación Primaria.

Tabla 3. Configuraciones cognitivas activadas por el grupo de profesores de primaria (A, B, C, D y E).

\begin{tabular}{|c|c|c|c|c|c|}
\hline Docente & Lenguaje & Definiciones & Propiedades & Procedimientos & Argumentos \\
\hline $\begin{array}{c}\text { Primaria- } \\
\text { A }\end{array}$ & $\begin{array}{c}\text { Número impar } \\
\text { Número par } \\
\text { Posibilidades } \\
\text { Caras } \\
\text { Porcentaje } \\
50 \% \\
1 / 2 \\
4 / 8\end{array}$ & $\begin{array}{l}\text { Probabilidad } \\
\text { simple } \\
\text { Espacio } \\
\text { muestral } \\
\text { Evento } \\
\text { Suceso }\end{array}$ & $\begin{array}{l}\text { Razón entre } \\
\text { el número de } \\
\text { ocurrencias } h \\
\text { de dicho } \\
\text { evento (casos } \\
\text { favorables) } \\
\text { y } \\
\text { el número tota } \\
\text { I de casos } \\
\text { posibles } n \text {. }\end{array}$ & $\begin{array}{l}\text { Aplicación de } \\
\text { definición de } \\
\text { Laplace: } \\
\text { Casos } \\
\text { favorables } \\
\text { sobre casos } \\
\text { posibles. }\end{array}$ & $\begin{array}{c}\text { Tesis: La probabilidad no } \\
\text { puede ser expresada por } \\
\text { medio de la fracción } 4 / 8 \\
\text { Razón: No, ya que hablamos } \\
\text { de } 6 \text { caras del dado con } 2 \\
\text { probabilidades. }\end{array}$ \\
\hline
\end{tabular}




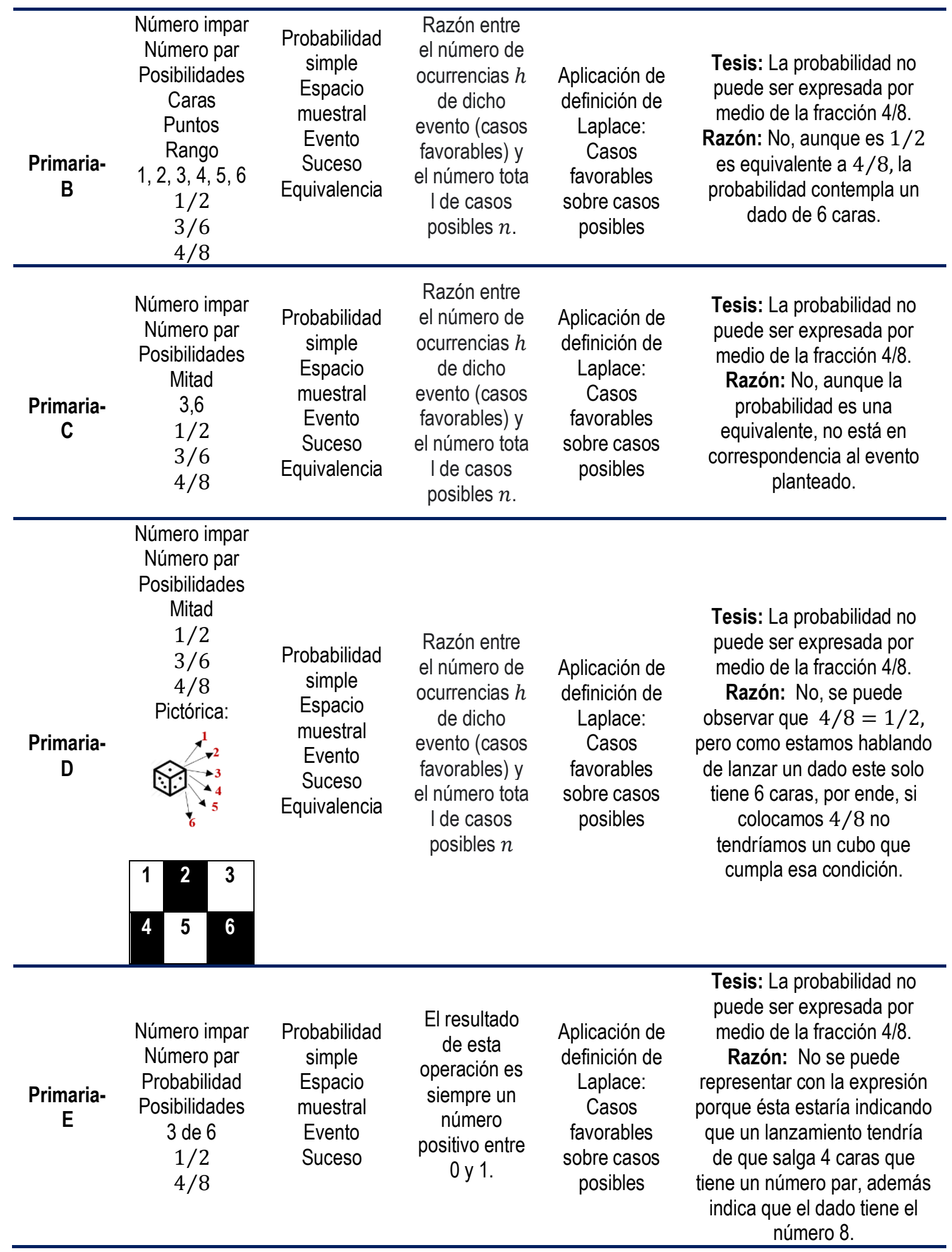

Fuente: Elaboración propia.

La Profesora [Primaria-A] calcula la probabilidad por medio de la expresión 50\%, manifiesta que el dado tiene 6 opciones que corresponde a todos los números que se puedan obtener (definición de la probabilidad simple); admite que la probabilidad puede ser representada por medio de la fracción $1 / 2$, ya que el dado tiene dos "probabilidades" (hace referencia a las opciones par e impar), pero ésta no puede ser expresada por medio de la fracción 4/8, en tanto, el dado 
tiene 6 caras. Por su parte, la Profesora [Primaria-B] representa la probabilidad por medio de la expresión $1 / 2$, alude que el dado tiene 6 caras, con un número en cada una de ellas ( 1 al 6 ) y 3 opciones posibles (los números pares 2, 4 y 6); reconoce que la probabilidad puede ser representada por la fracción $1 / 2$, puesto a que el dado tiene la mitad de los casos favorables y la fracción $3 / 6$ es equivalente a $1 / 2$, pero dicha probabilidad no puede ser representada por medio de la fracción 4/8, puesto que se habla de un dado de 6 caras.

La profesora [Primaria-C] representa la probabilidad por medio de la fracción 1/2, manifiesta que el dado tiene 6 caras, 3 son pares y corresponden a la mitad de posibilidades sobre el total, corrobora la equivalencia $3 / 6=1 / 2$, pero no admite que la probabilidad puede ser expresada por medio de la fracción 4/8, al respecto explícita que, si bien son muchas fracciones equivalentes a estas fracciones, la fracción $4 / 8$, no está en correspondencia con el evento planteado. La profesora [Primaria-D] calcula la probabilidad por medio de la expresión 3/6, alude que la probabilidad puede ser representada por medio de la fracción $1 / 2$; y si bien reconoce que $4 / 8=$ $1 / 2$, afirma que la fracción $4 / 8$ no puede representar la probabilidad pues no tendríamos un cubo que cumpla esa condición (haciendo referencia al dado de 6 caras).

Aunque la profesora [Primaria-E] no expresa la probabilidad por medio de una expresión matemática, específica la relación del número total de caras y la cantidad de números pares, identificando que de las 6 opciones del dado 3 cumplen la condición, admite que la probabilidad puede ser representada por medio de la expresión $1 / 2$, en tanto, el dado tiene dos opciones (pares-impares), pero no se puede representar con la expresión (se refiere a 4/8) porque estaría indicando que se tendría un dado con 4 caras pares y que el dado tiene el número 8.

Frente a las funciones semióticas las profesoras [primaria-A] y [primaria-E] establecen dos: la primera relaciona el antecedente "cálculo de la probabilidad al lanzar un dado y obtener un número par" con los consecuentes " $50 \%$ ", o "3 de 6"; la segunda entre "la probabilidad puede ser expresada por medio de la fracción 1/2" y "el dado tiene dos probabilidades (posibilidades)" o "se podría asumir el lanzamiento como el todo 1/2 representaría la probabilidad de que caigan números pares". Las profesoras [primaria-B], [primaria-C] y [primaria-D] establecen tres funciones semióticas: la primera, entre "cálculo de la probabilidad al lanzar un dado y obtener un número par" y " $3 / 6$ = 1/2"; "la expresión 1/2" o " 3/6"; la segunda, entre "la probabilidad puede ser expresada por medio de la fracción 1/2" y "los números pares, representa la mitad del total de números", "sí, 3 números de 6 posibles $3 / 6=1 / 2$, son equivalentes" o "todos miden igual"; y la tercera, entre "la probabilidad puede ser expresada por medio de la fracción 1/2" y "es 
equivalente a 1/2 ", "la representación de la mitad de los eventos posibles", "3/6 es equivalente a $1 / 2 "$

A continuación, se presenta el trabajo realizado por los profesores de secundaria $A, B, C, D$ y E, sobre la tarea del cálculo de probabilidad. Al igual que con el grupo de primaria, se seleccionan aquellos profesores que calculan correctamente la probabilidad, admiten que ésta puede ser representada por medio de la fracción $1 / 2$, reconocen que la expresión es equivalente a 4/8, pero no admite que esta última pueda representar la probabilidad pedida.

Tabla 4. Configuraciones cognitivas activadas por el grupo de profesores de secundaria.

\begin{tabular}{|c|c|c|c|c|c|}
\hline Docente & Lenguaje & Definiciones & Propiedades & Procedimientos & Argumentos \\
\hline $\begin{array}{c}\text { Secundaria - } \\
\text { A }\end{array}$ & $\begin{array}{l}\text { Número impar } \\
\text { Número par } \\
\text { Conjunto } \\
\text { Evento } \\
\text { Probabilidad } \\
\text { Posibilidades } \\
50 \% \\
0.5 \\
1 / 2 \\
4 / 8\end{array}$ & $\begin{array}{l}\text { Probabilidad } \\
\text { simple } \\
\text { Espacio } \\
\text { muestral } \\
\text { Evento } \\
\text { Suceso } \\
\text { Equivalencia }\end{array}$ & $\begin{array}{l}\text { El resultado de } \\
\text { esta operación } \\
\text { es siempre un } \\
\text { número positivo } \\
\text { entre } 0 \text { y } 1\end{array}$ & $\begin{array}{l}\text { Aplicación de } \\
\text { definición de } \\
\text { Laplace: Casos } \\
\text { favorables } \\
\text { sobre casos } \\
\text { posibles. }\end{array}$ & $\begin{array}{l}\text { Tesis: La probabilidad } \\
\text { no puede ser } \\
\text { expresada por medio } \\
\text { de la fracción 4/8. } \\
\text { Razón: No, representa } \\
\text { al mismo número, pero } \\
\text { no la misma situación, } \\
\text { en este caso el dado } \\
\text { tiene } 6 \text { posibilidades, } \\
\text { pero no se puede } \\
\text { generalizar para un } \\
\text { dado de } 8 \text { caras, etc. }\end{array}$ \\
\hline $\begin{array}{c}\text { Secundaria - } \\
\text { B }\end{array}$ & $\begin{array}{l}\text { Número impar } \\
\text { Número par } \\
\text { Conjunto } \\
\text { Evento } \\
\text { Probabilidad } \\
\text { Posibilidades } \\
50 \% \\
0.5 \\
3 / 6 \\
1 / 2 \\
4 / 8\end{array}$ & $\begin{array}{c}\text { Probabilidad } \\
\text { simple } \\
\text { Espacio } \\
\text { muestral } \\
\text { Evento } \\
\text { Suceso } \\
\text { Equivalencia }\end{array}$ & $\begin{array}{l}\text { El resultado de } \\
\text { esta operación } \\
\text { es siempre un } \\
\text { número positivo } \\
\text { entre } 0 \text { y } 1 .\end{array}$ & $\begin{array}{l}\text { Aplicación de } \\
\text { definición de } \\
\text { Laplace: Casos } \\
\text { favorables } \\
\text { sobre casos } \\
\text { posibles }\end{array}$ & $\begin{array}{l}\text { Tesis: La probabilidad } \\
\text { no puede ser } \\
\text { expresada por medio } \\
\text { de la fracción } 4 / 8 \text {. } \\
\text { Razón: Desde un punto } \\
\text { de vista matemático sí, } \\
\text { porque } \\
3 / 6=1 / 2=4 / 8, p \\
\text { ero desde un punto de } \\
\text { vista conceptual no. }\end{array}$ \\
\hline $\begin{array}{c}\text { Secundaria - } \\
\text { C }\end{array}$ & $\begin{array}{l}\text { Número impar } \\
\text { Número par } \\
\text { Conjunto } \\
\text { Evento } \\
\text { Probabilidad } \\
\text { Posibilidades } \\
\text { Intervalo } \\
{[0,1]} \\
\text { Equiprobable } \\
3 / 6 \\
1 / 2 \\
0.5 \\
4 / 8 \\
\end{array}$ & $\begin{array}{l}\text { Probabilidad } \\
\text { simple } \\
\text { Espacio } \\
\text { muestral } \\
\text { Evento } \\
\text { Suceso } \\
\text { Equivalencia }\end{array}$ & $\begin{array}{l}\text { El resultado de } \\
\text { esta operación } \\
\text { es siempre un } \\
\text { número positivo } \\
\text { entre } 0 \text { y } 1 .\end{array}$ & $\begin{array}{l}\text { Aplicación de } \\
\text { definición de } \\
\text { Laplace: Casos } \\
\text { favorables } \\
\text { sobre casos } \\
\text { posibles }\end{array}$ & $\begin{array}{l}\text { Tesis: La probabilidad } \\
\text { no puede ser } \\
\text { expresada por medio } \\
\text { de la fracción } 4 / 8 . \\
\text { Razón: No, aunque } \\
4 / 8=3 / 6=1 / 2 \\
\text { son equivalentes, esta } \\
\text { forma de presentar no } \\
\text { se ajusta directamente } \\
\text { con el problema. }\end{array}$ \\
\hline $\begin{array}{c}\text { Secundaria - } \\
\text { D }\end{array}$ & $\begin{array}{l}\text { Número impar } \\
\text { Número par } \\
\text { Evento } \\
\text { Probabilidad } \\
\text { Posibilidades } \\
3 / 6 \\
1 / 2\end{array}$ & $\begin{array}{l}\text { Probabilidad } \\
\text { simple } \\
\text { Espacio } \\
\text { muestral } \\
\text { Evento } \\
\text { Suceso } \\
\text { Equivalencia }\end{array}$ & $\begin{array}{l}\text { Razón entre } \\
\text { el número de } \\
\text { ocurrencias } h \\
\text { de dicho evento } \\
\text { [casos } \\
\text { favorables] y } \\
\text { el número total }\end{array}$ & $\begin{array}{l}\text { Aplicación de } \\
\text { definición de } \\
\text { Laplace: Casos } \\
\text { favorables } \\
\text { sobre casos } \\
\text { posibles }\end{array}$ & $\begin{array}{l}\text { Tesis: La probabilidad } \\
\text { no puede ser } \\
\text { expresada por medio } \\
\text { de la fracción } 4 / 8 . \\
\text { Razón: No, porque no } \\
\text { corresponde al mismo } \\
\text { evento de los dados. }\end{array}$ \\
\hline
\end{tabular}




\begin{tabular}{|c|c|c|c|c|c|}
\hline & $4 / 8$ & & $\begin{array}{c}\text { de casos } \\
\text { posibles } n .\end{array}$ & & \\
\hline $\begin{array}{c}\text { Secundaria - } \\
\text { E }\end{array}$ & $\begin{array}{c}\text { Número impar } \\
\text { Número par } \\
\text { Evento } \\
\text { Probabilidad } \\
\text { Posibilidades } \\
\text { Ocurrencia } \\
0.5 \\
3 / 6 \\
1 / 2 \\
4 / 8\end{array}$ & $\begin{array}{l}\text { Probabilidad } \\
\text { simple } \\
\text { Espacio } \\
\text { muestral } \\
\text { Evento } \\
\text { Suceso } \\
\text { Equivalencia }\end{array}$ & $\begin{array}{l}\text { El resultado de } \\
\text { esta operación } \\
\text { es siempre un } \\
\text { número positivo } \\
\text { entre } 0 \text { y } 1 .\end{array}$ & $\begin{array}{l}\text { Aplicación de } \\
\text { definición de } \\
\text { Laplace: Casos } \\
\text { favorables } \\
\text { sobre casos } \\
\text { posibles }\end{array}$ & $\begin{array}{l}\text { Tesis: La probabilidad } \\
\text { no puede ser } \\
\text { expresada por medio } \\
\text { de la fracción } 4 / 8 \text {. } \\
\text { Razón: No, porque no } \\
\text { corresponde al mismo } \\
\text { evento del dado. }\end{array}$ \\
\hline
\end{tabular}

Fuente: Elaboración propia.

El profesor [secundaria-A] calcula la probabilidad relacionando el número de casos posibles sobre el total de posibilidades, mediante la fracción 3/6, admite que la probabilidad puede ser representada por la fracción $1 / 2$, puesto que la probabilidad es un número que se obtiene del cociente entre dos números $a$ y $b$, pero no admite que la probabilidad pueda ser expresada por medio de la fracción $4 / 8$, plantea que si bien $3 / 6$ y 4/8, representan el mismo número, no representan la misma situación, en este caso el dado tiene 6 posibilidades. El profesor [Secundaria-B] establece el espacio muestral de las expresiones matemáticas $3 / 6=1 / 2=0.5=50 \%$, admite que la probabilidad puede ser representada por medio de la fracción $1 / 2$, debido a que las expresiones $1 / 2=0.5=50 \%$ (equivalencia sintáctica), pero no admite que la probabilidad pueda ser representada por medio de la fracción 4/8; aclara que si bien las expresiones 3/6 y 4/8, son iguales matemáticamente, "conceptualmente no" pues refieren situaciones diferentes (no reconoce equivalencia semántica).

El profesor [secundaria-C] enfatiza que se debe especificar si el dado es de 6 caras, puesto que todas las caras tienen la misma posibilidad de caer (equiprobable), partiendo de ello recurre a expresiones como $1 / 2,3 / 6$ y 0.5 , que representan los casos probables sobre el total de casos. Reconoce que las fracciones $1 / 2,3 / 6$ y $4 / 8$, son equivalentes, admiten que la probabilidad puede ser representada por medio de la expresión $1 / 2$, en tanto es una función donde el conjunto de llegada es el intervalo cerrado entre $[0,1]$ y la fracción $1 / 2$, se encuentra en dicho intervalo. Admite que la probabilidad puede ser representada por medio de la expresión $1 / 2$ (equivalencia sintáctica), pero desde el aspecto semántico el evento aleatorio no puede ser representado por medio de la fracción $4 / 8$, puesto que el dado tiene 6 caras.

El profesor [Secundaria-D] representa la probabilidad por medio de la fracción $1 / 2$, obtenida al simplificar 3/6; aunque reconoce que la fracción $4 / 8$, es equivalente a $1 / 2$ manifiesta que la probabilidad no puede ser expresada por medio de la fracción 4/8, debido que no corresponde al 
mismo evento de los dados. La profesora [Secundaria-E] representa la probabilidad por medio de las expresiones $3 / 6$ y 0.5 , obtenidas al considerar los casos favorables sobre los casos posibles (3 de 6 en el dado); reconoce que la probabilidad puede ser expresada por medio de la fracción $1 / 2$, puesto que al simplificar la expresión $3 / 6$ se obtiene $1 / 2$, que es igual a 0.5 . Pese a reconocer que la fracción $4 / 8$, es equivalente a $1 / 2$ y a 3/6, afirma que la expresión 4/8, no da cuenta de la probabilidad de ocurrencia de los números pares en el lanzamiento del dado, puesto que, el denominador 8 no hace referencia a las caras del dado, que son 6 .

Con relación a las funciones semióticas el profesor [Secundaria-D] establece dos; la primera, entre el antecedente "obtener un número par al lanzar un dado" y el consecuente " $1 / 2$, es equivalente a 3/6 "; y la segunda, "obtener un número par al lanzar un dado" y "3/6, 3 de 6 caras"; los profesores [secundaria-A], [secundaria-C] y [secundaria-E] establecen tres funciones semióticas: la primera, entre el antecedente "obtener un número par al lanzar un dado" y los consecuentes "casos posibles sobre el total de casos, 3/6" o "casos posibles sobre casos favorables"; la segunda, entre "obtener un número par al lanzar un dado" y "3/6 es equivalente a 1/2 " o "la expresión 3/6 es igual a 0.5"; la tercera, entre el antecedente "la probabilidad puede ser representada por medio de la expresión 1/2" y los consecuentes " $3 / 6$ es equivalente a $1 / 2$ y 50\%", "una función donde el conjunto de llegada es el intervalo cerrado entre $[0,1]$ y $1 / 2$ esta entre 0 y 1" o "al simplificar la expresión $1 / 2$ es igual a 0.5 "

El profesor [secundaria-B] establece cuatro funciones semióticas; la primera, entre el antecedente "obtener un número par al lanzar un dado" y el consecuente "casos posibles sobre el total de casos, 3/6"; la segunda, entre "obtener un número par al lanzar un dado" y "3/6 es equivalente a $1 / 2$ "; la tercera, entre el antecedente "obtener un número par al lanzar un dado" y el consecuente " $3 / 6$ es equivalente a $1 / 2$ y 0.5 "; y la cuarta entre "obtener un número par al lanzar un dado" y "3/6 es equivalente a 1/2, 0.5 y 50\%".

Como se muestra en las configuraciones cognitivas activadas por 10 profesores, reconocen que el cociente de las fracciones equivalentes es el mismo valor, en este caso 0.5 , otros que la $3 / 6$ es equivalente a expresiones $1 / 2,50 \%$ y 0.5 (equivalencia sintáctica), pero dotar de sentido estas expresiones plantean que el evento aleatorio no puede ser representado por medio de la fracción 4/8, debido que el dado no tiene 8 caras sino 6, es decir, no logran relacionar los sentidos asignados a estas fracciones (no reconocen la equivalencia semántica); aspecto que fue ampliado en las entrevistas semiestructuradas basadas en tareas. A continuación, se presenta la transcripción de una parte de una entrevista con el profesor [Secundaria- B]. 


\begin{tabular}{|c|c|c|c|}
\hline $\begin{array}{c}\text { Intervalo de } \\
\text { tiempo }\end{array}$ & Interlocutores & $\mathbf{N}^{\circ}$ & Diálogo \\
\hline$[9: 03-10: 05]$ & Entrevistadora & 1 & $\begin{array}{l}\text { Hablemos de la tarea que pide calcular la probabilidad que tiene } \\
\text { obtener un número par al lanzar un dado. Si esta puede ser } \\
\text { representada por medio de la fracción } 1 / 2 \text {, si esta podría ser } \\
\text { expresada por medio de la fracción } 4 / 8 \text { (señalando la pantalla con el } \\
\text { cursor, muestra las respuestas dadas por la profesora). Me gustaría } \\
\text { que me comentara cómo se dio cuenta que desde el punto de vista } \\
\text { matemático las fracciones } 1 / 2 \text { y } 4 / 8 \text {, son equivalentes, pero la } \\
\text { fracción } 4 / 8 \text {, no representa la probabilidad pedida. }\end{array}$ \\
\hline
\end{tabular}

[10:05 - 11:06] Secundaria-B 2 Bueno, en realidad uno debe ser capaz de modelar una expresión matemática cuando son equivalentes porque son iguales, pero en cuestiones de probabilidad la expresión matemática debe modelar el evento aleatorio, por ejemplo, en el caso del dado se tiene 6 caras y 3 de ellas son pares, la parte conceptual sería diferente porque se perdería la esencia de la situación.

\begin{tabular}{cccl}
\hline$[11: 06-11: 46]$ & Entrevistadora & 3 & Me gustaría que me explicara el por qué se perdería la esencia. \\
\hline$[11: 46-12: 28]$ & Secundaria-B & 4 & $\begin{array}{l}\text { Porque ya no se está hablando de un dado de } 6 \text { caras o se estaría } \\
\text { representando las dos opciones del dado [pares e impares] y uno con } \\
\text { los estudiantes debe ser muy claro para que no se confundan. }\end{array}$ \\
\hline$[12: 28-13: 14]$ & Entrevistadora & 5 & $\begin{array}{l}\text { Y si tenemos un dado de ocho caras, ¿sí podría expresar la } \\
\text { probabilidad con la fracción 4/8? }\end{array}$ \\
\hline$[13: 14-14: 01]$ & Secundaria-B & 6 & $\begin{array}{l}\text { Claro, ahí sí serviría, porque los } 4 / 8 \text { refiere a la misma situación de } \\
\text { marcar números pares en un dado de ocho caras. }\end{array}$ \\
\hline
\end{tabular}

En el fragmento 2 de la anterior transcripción el profesor reconoce que las fracciones $1 / 2$, $3 / 6$ y $4 / 8$, son equivalentes en tanto representan el mismo número, alude que en cuestiones de la probabilidad no todas las fracciones son pertinentes para modelar el evento aleatorio en cuestión, puesto que, la expresión debe explicitar simbólicamente la situación, en este caso la expresión 4/8, no permite modelar el evento aleatorio, puesto que esta fracción pierde la esencia que ofrece las fracciones $1 / 2$ y $3 / 6$, las cuales describen alguna de las características del evento aleatorio, hecho que no permite articular los sentidos asignados a tales expresiones; en el caso que el objeto físico, "el dado", tenga 8 caras dicha fracción sería pertinente, como lo explicita en los fragmentos 4 y 6 .

En conclusión, los profesores reconocen desde el aspecto sintáctico la equivalencia entre las expresiones, pero dotar de sentido y significado las expresiones matemáticas que modelan el evento aleatorio enmarcado en la tarea manifiestan que no corresponde al mismo evento del dado, o no corresponde a la misma situación, puesto que el dado tiene 6 posibilidades y no 8 (no reconoce equivalencia semántica). Hecho que deja en evidencia un reconocimiento explícito de la equivalencia sintáctica entre las fracciones, pero un "anclaje" al objeto físico, delimitado por el dado (número de caras, y los casos favorables), lo cual no permite articular los sentidos asignados a dichas expresiones, en tanto no aceptan que la fracción $4 / 8$, sea representativa de dicha 
probabilidad. A continuación, se resaltan algunos rasgos característicos sobre la manera de calcular la probabilidad por parte de los profesores de primaria, secundaria y estudiantes, así como las dificultades que encuentran éstos para relacionar los sentidos asignados entre sí.

Tabla 5. Similitudes y diferencias entre las soluciones dadas por los profesores de primaria, secundaria y estudiantes frente al cálculo de la probabilidad.

\section{Profesores de primaria}

Algunos profesores calculan la probabilidad por Figura 4. ¿Cuál es la probabilidad de que lanzando un dado medio del registro semiótico en lenguaje natural, identifican el número total de caras y la cantidad de números pares, $(6$ opciones del dado, 3 cumplen la condición). Tal y como se evidencia en la Figura 4 que corresponde al argumento dado por la profesora de primaria-E. Otros realizan la representación icónica del dado y las profesoras A, B, C y D lo hacen por medio de las expresiones $3 / 6,1 / 2,50 \%, 0.5$

se obtenga un número par?

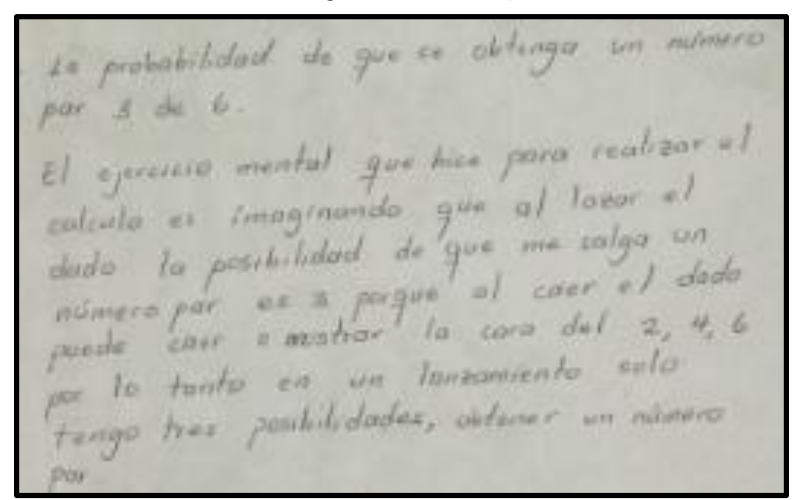

Fuente: Producción realizada por la profesora de primaria $-\mathrm{E}$.

\section{Profesores de secundaria}

Figura 5. ¿La anterior probabilidad se podría representar con la expresión $1 / 2$ ?

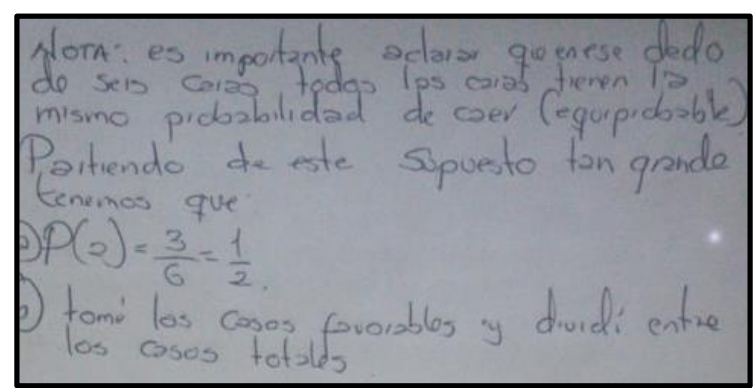

Los profesores de secundaria $A, B, C, D$ y $E$, recurren a una, dos tres o cuatro expresiones matemáticas ya sea $3 / 6,1 / 2,50 \%, 0.5$, a diferencia del grupo de profesores de primaria no expresan la probabilidad por medio de lenguaje natural.

Fuente: Producción realizada por la profesora de secundaria- $\mathrm{C}$.

\section{Estudiantes}

Rojas (2012) plantea que los estudiantes calculan la probabilidad por medio de las expresiones numéricas $50 \%$ y $3 / 6$, alude que reconocen la equivalencia entre las fracciones $3 / 6$ y $1 / 2$. Frente a las funciones semióticas manifiesta que en su mayoría los estudiantes establecen 3; una entre el antecedente "la probabilidad que al lanzar un dado se obtenga un número par" y el consecuente "la probabilidad es 3/6"; otra entre "la probabilidad que al lanzar un dado se obtenga un número par" y "la probabilidad es $1 / 2$ "; y la tercera, entre el antecedente "la probabilidad que al lanzar un dado se obtenga un número par" y el consecuente "la probabilidad es 50\%".

\section{Articulación semiótica}

Los profesores reconocen sintácticamente la equivalencia de las expresiones $3 / 6,1 / 2$ y 4/8, pero semánticamente la probabilidad no puede ser representada mediante la fracción $4 / 8$, puesto que, no refiere el mismo evento aleatorio, puesto que, el dado no tiene 8 caras, aspecto que impide que se articule los sentidos asignados a estas. Al igual que las soluciones dadas por los estudiantes reconocen la equivalencia entre las fracciones $3 / 6,1 / 2$ y $4 / 8$, pero no logran relacionar los sentidos asignados a estas expresiones matemáticas, en tanto relacionan la expresión $4 / 8$, con otro evento aleatorio; 
resultados que muestran que no existen diferencias entre estudiantes, profesores de primaria y secundaria; tal y como se muestra en las Figuras 6 y 7.

Figura 6. ¿La probabilidad se podría representar con la expresión 4/8?

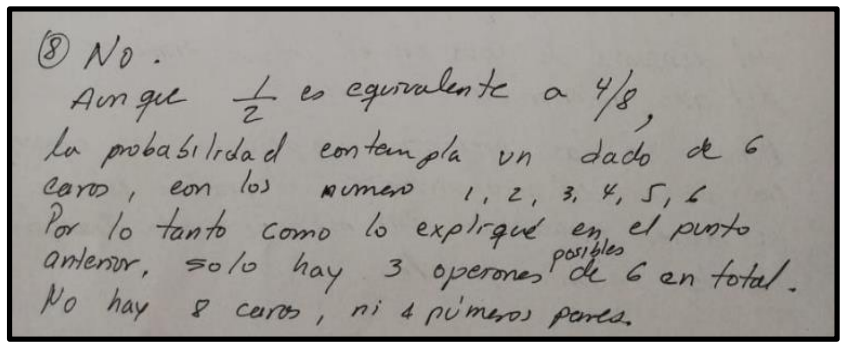

Fuente: Producción realizada por la profesora de primaria-B.

Figura 7. ¿La probabilidad se podría representar con la expresión $\mathbf{4 / 8}$ ?

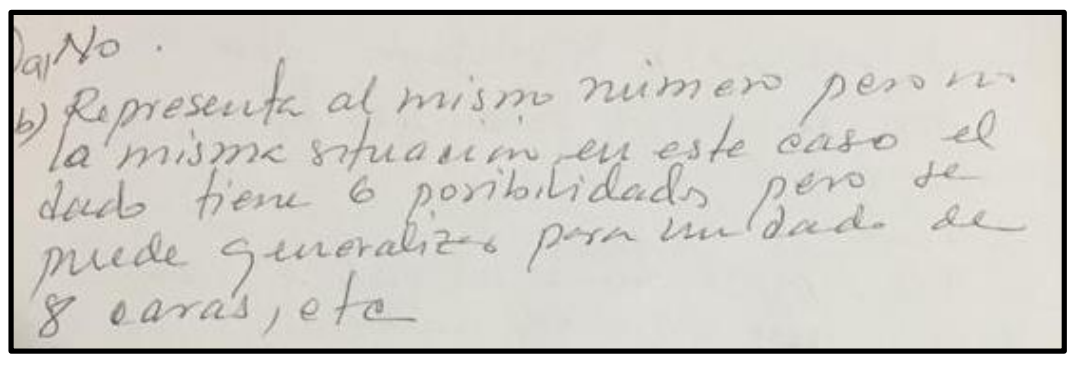

Fuente: Producción realizada por el profesor de secundaria-A.

Fuente: Elaboración propia.

De manera general las producciones muestran que los 62 profesores recurren a la definición de la probabilidad simple "casos favorables sobre posible", con base a esta definición representan la probabilidad por medio de expresiones matemáticas como: 3/6,1/2, $0.5050 \%$, la diferencia radica en la manera de expresar la probabilidad del evento aleatorio; los profesores de primaria, en su mayoría recurren a un lenguaje natural, mientras los profesores de secundaria expresan el cálculo de la probabilidad haciendo uso de diferentes expresiones numéricas equivalentes.

En conclusión, a partir de las producciones de los profesores de matemáticas en la tarea que les fue propuesta, se evidencian similitudes con respecto a las dificultades que encuentran los estudiantes para articular sentidos asignados a representaciones semióticas obtenidas mediante tratamiento. En los resultados que reporta Rojas (2012) sobre la manera en que los estudiantes calculan la probabilidad y las dificultades que encuentran estos para relacionar los sentidos asignados a las fracciones $3 / 6,1 / 2$ y $4 / 8$ como representativas de la probabilidad pedida, plantea que, si bien ellos admiten la equivalencia (sintáctica) entre las fracciones la razón fundamental que dificulta la articulación semiótica radica en la permanencia del objeto físico referido en la tarea planteada, el dado con sus 6 caras (no 8), dificultad denominada por este autor "anclaje a la situación", pues se evidencia que efectivamente el sentido asignado a la fracción 4/8 está "anclado" al objeto físico "dado de seis caras". 
Frente al anclaje, Rojas (2012) muestra evidencias de estudiantes que argumentan: "el tema principal es el dado y éste sólo tiene 6 caras, entonces 4/8 pues no, no es tan representativo del dado, no tiene ocho caras, ni cuatro números pares", "hay 3 opciones de 6 y que no puede ser, ni de las 4 ni tampoco puede ser de 8", "la fracción 4/8, estaría mal planteada pues el problema debe resolverse con base en las caras del dado (y éste) nunca va a tener ocho caras", "la fracción 4/8 no puede ser la probabilidad de obtener un número par al lanzar un dado, ya que si el dado tiene 6 caras, hay 3 opciones de 6 , entonces no puede tomarse el número de referencia cuatro ni ocho".

Los anteriores argumentos son similares a los planteados por los profesores de matemáticas en las producciones realizadas con respecto a la misma tarea, quienes reconocen la equivalencia sintáctica de las expresiones, pero plantean que la probabilidad no puede ser representada por medio de la fracción $4 / 8$, en tanto "el dado no tiene 8 caras", o, "en un dado tradicional la fracción $3 / 6$, muestra en detalle las 3 caras que hacen referencia a los números pares y el 6 a las caras del dado, en cambio en 4/8, eso no estaría claro"; en este caso se evidencia que los profesores establecen la probabilidad en términos al evento aleatorio y existe un "anclaje a la situación", que les impide realizar las articulaciones requeridas.

\section{Conclusiones}

El trabajo realizado por el grupo de profesores que conforman el estudio de caso puso en evidencia dificultades que algunos de ellos encuentran para articular sentidos asignados a expresiones matemáticas obtenidas mediante tratamiento. En el apartado de los resultados se presentaron evidencias de que, si bien los profesores reconocen la equivalencia sintáctica entre dos o más expresiones, no siempre logran articular los sentidos asignados a dichas expresiones y éstas son asociadas con objetos matemáticos o situaciones diferentes, en este caso eventos aleatorios diferentes (no reconocen equivalencia semántica). Estos resultados corroboran que, para el caso de los profesores de matemáticas, no solo las transformaciones semióticas de conversión se relacionan con dificultades para la comprensión de un objeto matemático, sino también las transformaciones de tratamiento (D'AMORE 2006; SANTI 2011; ROJAS 2012).

Por otra parte, se realizó un análisis de las producciones realizadas por los profesores a la luz de las dificultades que encuentran los estudiantes para articular los sentidos asignados a representaciones semióticas obtenidas mediante tratamiento reportadas en la literatura, con el fin de establecer similitudes y diferencias entre las dificultades que encuentran los profesores de matemáticas. Específicamente los resultados obtenidos en este estudio se contrastaron con los 
hallazgos reportados por Rojas (2012), quien clasificó en cuatro grupos las dificultades que encuentran los estudiantes: i) reconocimiento icónico de las expresiones; ii) anclaje a situaciones dadas; iii) interacción y cambios en la interpretación; y iv) dificultades con el lenguaje matemático. Se encontró evidencias de dificultades similares en el trabajo con profesores de matemáticas. En el presente documento se reportan específicamente las relacionadas con el "anclaje a situaciones".

Los resultados mostraron que, cuando en la tarea se pregunta si 4/8 representa la "probabilidad de que al lanzar un dado se obtenga un número par", los profesores primaria-A, primaria- $B$, primaria- $C$, primaria- $D$, primaria- $E$, secundaria- $A$, secundaria- $B$; secundaria-C, secundaria-D y secundaria-E reconocen la equivalencia sintáctica entre las fracciones $1 / 2,3 / 6$ y 4/8, pero el "anclaje a la situación" les impide reconocer la equivalencia semántica. Por ejemplo, la profesora de primaria-A afirma: "yo la verdad me centré en el evento aleatorio que se enmarca en la tarea (un dado de 6 caras), entonces el tener 4/8, desde mi perspectiva no cumple con las 6 caras, ahora en el caso de $1 / 2$, sí se cumple porque tenemos dos opciones (paresimpares) en cambio en cuatro octavos a mí me parece que no se puede decir cuatro de las ocho caras, por ejemplo, porque el dado no tiene 8 caras"; o el profesor secundaria-A quien afirma: "sí, aunque son fracciones equivalentes las fracciones representa casos diferentes, en un dado tradicional es lógico decir 3 de 6, pero no se puede decir 4 de 8 , aunque son expresiones equivalentes porque representa $1 / 2$, pero cada uno representa situaciones diferentes pues el dado tiene 6 caras". Estos argumentos son similares a los planteados por los estudiantes al resolver la misma tarea, quienes manifestaron que la fracción 4/8, no representa la probabilidad pedida, puesto que ésta no es "representativa del dado", o que: " $4 / 8$, no es tan representativo del dado, ya que el dado no tiene ni ocho caras, ni cuatro números pares" (ROJAS, 2012, p. 120).

Otros estudiantes argumentaron que la fracción 4/8 está "mal planteada" pues el dado no tiene 8 caras, al respecto aluden: "la fracción estaría mal planteada, para (...) resolver el problema, en base de las caras del dado (...) Un dado nunca va a tener ocho caras". Aunque algunos estudiantes aceptan que las fracciones $4 / 8$ y $3 / 6$, son iguales, en tanto ambos equivalen a la mitad, consideran que esta fracción no permite tener "precisión", ni ser claros en el evento aleatorio, pues el dado tiene sólo 6 caras. Algunos expresaron que: "la fracción estaría mal planteada, para (...) resolver el problema, con base a las caras del dado (...) Un dado nunca va a tener ocho caras" (ROJAS, 2012, p. 121); argumentos que dan cuenta de la dificultad planteada y de la similitud con los planteados por los profesores de matemáticas.

En relación con la complejidad de la noción de equivalencia, los profesores realizan una serie de prácticas, como el cálculo de la probabilidad a partir de la regla de Laplace, relacionan la 
equiprobabilidad de todos los resultados de un experimento aleatorio, definida como el cociente entre el número de resultados favorables a que ocurra el suceso y el número de resultados posibles del experimento, así como la simplificación de expresiones que permite corroborar la equivalencia entre las expresiones $1 / 2,3 / 6,4 / 8,0.5$ y $50 \%$ (significado parcial sintáctico); sin embargo, varios de ellos no admiten la fracción 4/8 como representante de dicha probabilidad, consideran que "no es representativa del dado", puesto que, el dado no tiene 8 caras, ni 4 caras favorables (significado parcial semántico). Se confirma lo planteado por Chalé-Can et al. (2017) sobre la necesidad de reconocer aspectos sintácticos y semánticos de la equivalencia, así como de su necesaria articulación, en tanto cada significado posibilita prácticas matemáticas específicas.

\section{Referencias}

CHALÉ-CAN, Sergio. Aspectos sintácticos y semánticos de la equivalencia de expresiones algebraicas en secuencias visuales. 2018. Tesis (Doctorado en Ciencias, especialidad en Educación Matemática). CINVESTAV -IPN, México.

CHALÉ-CAN, Sergio; FONT, Vicenç; ACUÑA, Claudia. La semántica y la sintáctica en la equivalencia de expresiones algebraicas. En CONTRERAS, J. M.; ARTEAGA, P.; CAÑADAS, G. R.; GEA, M. M.; GIACOMORE, B.; LÓPEZ-MARTÍN, M. M. (Eds.), Actas del Segundo Congreso International Virtual sobre el Enfoque Ontosemiótico del Conocimiento y la Instrucción Matemáticos. p. 1-10, 2017.

D'AMORE, Bruno. Objetos, significados, representaciones semióticas y sentido. Relime, número especial. p. 177-195, 2006.

DISTÉFANO, María Laura; AZNAR, María Andrea; POCHULU, Marcel David. Errores asociados a la representación geométrica-vectorial de números complejos: un análisis ontosemiótico. UNIÓN. Revista Iberoamericana de Educación Matemática. p. 61-80, 2012.

DISTÉFANO, María Laura; POCHULU, Marcel David; FONT, Vicenç. Análisis de la complejidad cognitiva en la lectura y escritura de expresiones simbólicas matemáticas. Journal of Research in Mathematics Education, v. 4, n. 3, p. 202-233, 2012.

DISTÉFANO, María Laura; POCHULU, Marcel David. Trama de funciones semióticas en actividades de simbolización. En J. M. Contreras, P. Arteaga, G. R. Cañadas, M. M. Gea, B. Giacomone y M. M. López-Martín (Eds.). Actas del Segundo Congreso International Virtual sobre el Enfoque Ontosemiótico del Conocimiento y la Instrucción Matemáticos. 2017.

DUVAL, Reymond. Semiosis y pensamiento humano: Registros semióticos y aprendizajes intelectuales (M. Vega, Trad.). Cali: Universidad del Valle. 2004.

FONT, Vicenç; GODINO, Juan. D; GALLARDO, Jesús. La emergencia de los objetos a partir de las prácticas matemáticas. Educ Stud Math, v. 82, p. 97-124, 2012.

GODINO, Juan D. Studying the median: A framework to analyse instructional processes in Statistics Education. In PHILLIPS, B. (Ed.), Proceedings of the ICOTS-6. CDROM. IASE, 2002. 
GODINO, Juan D; BATANERO, Carmen; FONT, Vicenç. The ontosemiotic approach to research in mathematics education. ZDM. The International Journal on Mathematics Education, 39 (12), 127-135, 2007.

GODINO, Juan D; CASTRO, Walter; AKE, Lilia; WILHELMI, Miguel. Naturaleza del razonamiento algebraico elemental. Bolema, vol.26, n.42b, p. 483-512, 2012.

GODINO, Juan D; BATANERO, Carmen; BURGOS, María y GEA, María M. Una perspectiva ontosemiótica de los problemas y métodos de investigación en educación matemática. Revemop, v.3, p. 1-30, 2021. DOI: https://doi.org/10.33532/revemop.e202001

KIERAN, Carolyn; SALDANHA, Luis. Computer algebra systems (CAS) as a tool for coaxing the emergence of reasoning about equivalence of algebraic expressions. En H. L. Chick y J. L. Vincent (Eds.) Proceedings of the 29th. Conference of the International Group for the Psychology of Mathematics Education, v. 3. Melbourne: PME, p. 193-200, 2005.

KIERAN, Carolyn; BOILEAU, André; TANGUAY, Denis; DRIJVERS, Paul. Desing researches' documentational genesis in a study on equivalence of algebraic expressions. The International Journal on Mathematics Education, v. 45, p. 1045-1056, 2013.

MEJÍAS, Cristian. Evaluación de los conocimientos para la enseñanza del álgebra en profesores en ejercicio de educación primaria. 2019. Tesis (Doctorado en Educación). Universidad de Girona, Girona, España.

RADFORD, Luis. Syntax and Meaning. In HøINES, M. J.; FUGLESTAD, A. B. (Eds.), Proceedings of the 28 Conference of the international group for the psychology of mathematics education (PME 28), v. 1. Norway: Bergen University College, p. 161-166, 2004.

ROJAS, Pedro. Articulación y cambios de sentido en situaciones de tratamiento de representaciones simbólicas de objetos matemáticos. 2012. 128f. Tesis (Doctorado en Educación). Universidad Distrital Francisco José de Caldas, Bogotá, Colombia

ROJAS, Pedro. Objetos matemáticos, representaciones semióticas y sentidos. Enseñanza de las Ciencias. v. 33, n. 1, p. $151-165,2015$. DOI: https://doi.org/10.5565/rev/ensciencias. 1479

SOLARES, Armando. y KIERAN, Carolyn. Articulating syntactic and numeric perspectives on equivalence: the case of rational expressions. Educational Studies in Mathematics. v. 42, p. 115148, 2013.

SANTI, Georgio. Objectification and semiotic function. Educational. Studies Mathematics, 77, p. 285-311, 2011.

STAKE, Robert. Case Study, en DENZIN, N.K.; LINCOLN, Y.S. (Eds.). Handbook of Qualitative Research. London: Sage, p. 236-247, 1994. 